\section{Edycaçäa

ARTIGO

\section{Editores}

Maria Inês Côrte Vitoria

PUCRS, RS, Brasil

Pricila Kohls dos Santos

PUCRS, RS, Brasil

\section{Equipe Editorial}

Rosa Maria Rigo

PUCRS, RS, Brasil

\title{
Estrategias de intervención en conductas disruptivas
}

\author{
Intervention Strategies in disruptive behavior
}

María del Carmen Gómeza ${ }^{\mathrm{a}}$, Alejandrina da Resurrección Cuña ${ }^{\mathrm{b}}$

\section{RESUMEN}

Las conductas disruptivas son un tema preocupante, para los profesionales de la enseñanza. Por este motivo hemos decidido elaborar un trabajo en el que profundizar sobre las mismas; veremos por lo tanto qué significan las conductas disruptivas y cuáles son los factores que las provocan. También conoceremos estrategias para tratar las que conductas disruptivas; por ello se ha hecho una selección de técnicas de modificación de conducta muy útiles para estos casos.

Palabras clave: Conducta; Disrupción; Modificación; Técnicas; Educación.

\section{ABSTRACT}

Disruptive behavior in a concern for teaching professionals, since this type of behavior in many cases. That's why we decided to develop this work where we will delve on disruptive behavior; therefore we see why disruptive behavior and the factors causing the same means. Also we know the strategies to address that disruptive behavior; therefore we have made a selection of modification techniques useful behavior for these cases.

Keywords: Conduct; Disruption; Modification; Technical; Education.

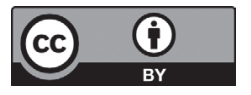

Este artigo está licenciado sob forma de uma licença Creative Commons Atribuição 4.0 Internacional, que permite uso irrestrito, distribuição e reproduçāo
em qualquer meio, desde que a publicaçăo original seja corretamente citada. http://creativecommons.org/licenses/by/4.0/deed.pt BP a Universidad Católica de Ávila. Diplomada en educación especial, en audición y lenguaje, en educación infantil. Licenciada en Pedagogía. Doctora en Pedagogía por la Universidad Pontificia de Salamanca. Actualmente soy profesora responsable en la E.U. Fray Luis de las asignaturas de didáctica general y teoría e historia de la educación.

b E.U. Fray Luis de león. Universidad Católica de Ávila. Grado en educación primaria, mención de educación especial. 


\section{Introducción}

T as conductas disruptivas son un tema de gran importancia para los profesionales de la educación, ya que afectan directamente al proceso de enseñanza-aprendizaje.

Cada vez es más común escuchar el término de conductas disruptivas; se aplica para referirseal niño que es violento, al que es agresivo, al que no obedece, al que no acata las normas de clase, al que habla en clase, al que molesta a los compañeros, etc. Se utiliza el termino para definir cualquier mal comportamiento del niño.

Para comprender y delimitar lo qué es una conducta disruptiva vamos a analizar las definiciones que de ello hacen diferentes autores:

Según García Correa (2008)

Entendemos por conducta disruptiva aquella que influye negativamente en el proceso docente y/o supone un grave trastorno para el normal desarrollo de la vida escolar. Es aquella conducta que genera conflictos en el aula (p.37).

Para Uruñuela (2006)

El término disrupción se refiere las conductas que llevan a cabo aquellos alumnos dentro de las clases que puedan buscar diferentes objetivos como llamar la atención, reclamar su deficiente historia académica, pero que tienen como consecuencia que el profesorado no pueda llevar a cabo de manera adecuada su tarea profesional de enseña, impidiéndole que pueda hacer la explicación de los temas realizar las actividades oportunas o aplicar las evaluaciones oportunas que considere necesarias (p. 5).

Así, podemos decir que una conducta disruptiva es aquel comportamiento del alumno que interfiere, molesta, interrumpe e impide que el docente lleve a cabo su labor educativa. Estas conductas además de perjudicar el desarrollo de la función docente, también provocan un mal ambiente y malestar en el aula. El proceso de enseñanzaaprendizaje se verá dañado por estas conductas y como consecuencia el alumno no alzará los objetivos educativos planteados. 


\section{Conductas disruptivas}

\subsection{Conductas disruptivas más frecuentes}

A continuación veremos diferentes conductas-problema que nos indican diferentes autores siguiendo a Vallés (2002). Hollins, en el año 1955 elaboró una lista de conductas-problema como son:

- Atraer la atención.

- Malos modales.

- Amenazas, crueldades.

- Falta de cuidados.

- Mentir.

- Cobardía.

- Ensueños.

- Falso, astuto, acusica.

- Desobediencia.

- Riñas, uso habitual de la violencia.

- Falta de atención.

- Insolencia, falta de respeto.

- Falta de concentración.

- Falta habitual de concentración.

- Falta habitual de puntualidad.

- Vagancia, gandulería.

En España Fernández Pozar (2002) redacta otra lista de conductas problema basadas en la escala de Hollins, en las que las conductas son consideradas como (muy grave, grave, poca grave, nada grave) así como una clasificación frecuencial (máxima, regular, poco). En 1979 Pelechanose enumeran otras conductas-problema en el aula:

- Golpear a los compañeros.

- Ser violentos.

- Buscar peleas.

- Romper cosas.

- Molestar a los compañeros mientras trabajan.

- Amenazar. 
- Intimidar a los demás.

- Plantar cara y adoptar una postura desafiante ante el profesor.

- Tener problemas con los demás niños al poco tiempo de hablar con ellos.

- Burlarse de sus compañeros cuando hacen algo mal o sufren alguna inferioridad.

- Irritable.

- Imponer reglas del juego en beneficio propio.

- Físicamente deprimidos.

- Nervioso, tembloroso, lívido, intranquilo cuando espera decir la lección.

Se puede comprobar que muchas de las conductas que destaca Hollins son comunes a las de Pelechano. En España se llevaron a cabo investigaciones sobre conductas disruptivas que dejaron conclusiones como que:

Ordinariamente los alumnos problemáticos presentan más de una conducta distorsionadora en el aula, el promedio de alumnos disruptivos por clase es de 3-4 teniendo la clase unos 30 alumnos, hay desproporción entre los colegios, respecto a la hora de producirse es indistintamente y las conductas más frecuentes son: charlatán, distraído, inquieto, agresivo, ruidoso, injurioso, rebelde, indolente, mentiroso, no participativo (GARCÍA CORREA y otros, 1983, p.38, en GARCÍA, 2008).

Gotzens, C. (1986) tomando como punto de partida un análisis de varios trabajos señala las siguientes conductas distorsionadoras:

- Motrices: estar fuera del asiento, dar vueltas por la clase, saltar, andar a la "pata coja", desplazar la silla, ponerse de rodilla sobre la misma, balancearse.

- Ruidosas: golpear el suelo con los pies y con las manos los asientos, dar patada a la silla o a la mesa, dar palmadas, hacer ruido con el papel, rasgar papel, tirar libros u objetos, derribar las sillas o mesas.

- Verbales: conversar con otros, llamar al profesor para conseguir la atención, gritar, cantar, silbar, reír, toser, llorar.

- Agresivas: pegar, empujar, pellizcar, abofetear, golpear con objetos, arrebatar objetos o trabajos pertenecientes a otros, destrozar la propiedad ajena, lanzar objetos.

- De orientación en la clase: volver la cabeza y/o el cuerpo hacia otro compañero, mostrar objetos a otro, observar a otros largamente en el tiempo.

\subsection{Conductas disruptivas y niveles educativos}

Dependiendo de la etapa educativa se manifiestas distintas conductas disruptivas, así siguiendo a Correa (2008) tenemos que las más frecuentes según la etapa son: 
Tabla 1. Conductas disruptivas clasificadas por edad.

\begin{tabular}{lll}
\hline Etapa de infantil & Etapa de primaria & Etapa de secundaria \\
Estar fuera del sitio & Ruidosas & Verbales \\
Saltar & Verbales & Agresivas \\
Dar vueltas por la clase & Agresivas & Injuriosas \\
Interrumpir & & Rebeldes \\
Pellizcar & & \\
Destrozar y golpear objetos & & \\
\hline
\end{tabular}

Las conductas disruptivas en el aula de infantil se deben generalmente a que aún él niño no ha interiorizado las normas. Al final de la etapa de primaria y secundaria las razones son entre otras; aburrimiento en el aula, desinterés por los temas dados en el aula, problemas emocionales, molestar al profesor, etc.

\section{Factores que intervienen en las conductas disruptivas}

Los factores o causas que generan las conductas disruptivas en el aula son de naturaleza variada. Algunos investigadores defienden la idea de que las causas son principalmente ajenas al ámbito educativo, mientras que otros creen que las conductas disruptivas están relacionadas con ámbito educativo. Es una cuestión bastante compleja, por lo que no podemos señalar que la conducta disruptiva está en una sola causa, más bien debemos ver todas las causas posibles para llegar a comprender realmente cuales son las razones por las que un niño tiene un comportamiento inapropiado en el aula, que perjudica el desarrollo del proceso enseñanza-aprendizaje. Con respecto a esto Uruñuela (2006) afirma que:

El análisis de la disrupción debe plantearse desde la complejidad, y no desde la simplicidad; muchas veces se tiene la tentación de buscar causas y factores simples, una causa única, pensando que de esa forma es posible comprender y explicar lo que está pasando en los centros en materia de disciplina; y si a ello se une la propensión a buscar causas de los problemas del centro en factores ajenos al mismo, atribuyendo a los propios alumnos, a sus padres, a los medios de comunicación o a la sociedad moderna en general la responsabilidad de los problemas de conductas, es entonces muy fácil que nos quedemos en la superficie y apenas se logre una comprensión y explicación de cómo transcurre la convivencia en los centros educativos (p. 19). 
Teniendo en cuenta lo que nos dice Uruñuela, para conocer las causas de las conductas disruptivas de los niños debemos conocer los factores tanto externos al centro como internos del ámbito escolar, ya que eso nos ayudará a abordar la problemática de las conductas de una manera mucho más profunda y real.

Los factores externos son aquellos que causan conductas disruptivas y no están relacionados con el centro escolar; sin embargo no debemos olvidar que existen otros factores, los factores internos del centro. Estos últimos, son los que se dan dentro del aula y causan conductas disruptivas en el interior de la misma. Pueden ser modificados por el profesor y junto con los factores externos del alumno son los causantes, la mayor parte de las veces, de que los alumnos adopten conductas disruptivas. A continuación y siguiendo a Uruñuela presentaremos algunos factores internos que pueden producir conductas disruptivas:

- Organización del aula y del centro: En relación a esto, en algunos centros, se distribuye a los alumnos en grupos rígidos e inflexibles, favoreciéndose así este tipo de conductas.

- Metodología del docente: La mayoría son clases expositivas donde el alumno se limita a escuchar mientras elprofesor habla, es decir, el alumno no es el centro de la clase sino el profesor.

- Falta de interacción entre alumno-profesor: El profesor debe entablar cierta relación con el alumno, preguntarle ¿qué tal esta? ¿si entiende la asignatura?

\section{Elementos preventivos de las conductas disruptivas}

\subsection{La gestión de aula y el estilo docente}

La relación que exista entre alumno-maestro es fundamental, ya que esto puede favorecer un buen clima en el aula y evitar así conductas disruptivas. El maestro es quién explica los contenidos, decide la metodología, la tipología de actividades que se van a llevar a cabo, organiza el tiempo y espacio; es pues, el papel del maestro en el aula fundamental. Además de todo esto, el docente, también debe poner orden en el aula,fundamentalpara evitar cualquier conductas disruptivas. Que los alumnos sepan que el aula es un lugar de orden donde se debe trabajar y no un sitio de recreo donde cada uno hace los que quiere, es tarea del maestro.

En cuanto al estilo docente pueden existir tantos como maestros hay, ya que cada uno tiene sus propias características y maneras de desarrollar su trabajo. Ningún estilo es mejor que otro, simplemente es necesario usar aquel que se adapte al grupo que tengamos.

Torrego y Fernández, en su artículo "Protocolo de actuación ante la disrupción en el aula" (p. 105) recogen algunas propuestas de gestión y manejo de la interacción en el aula: 
- Efectuar gestos y miradas que indiquen la conducta deseada.

- Realizar indicaciones centradas en la tarea, no tanto en la persona.

- Silencio del profesor ante la dispersión del aula.

- Invadir territorio, acercamiento a la zona o alumno disruptivo.

- Llamar la atención de forma seria y breve.

- Utilizar el humor, no el sarcasmo.

- Mensaje en primera persona.

- Explicitar de forma breve las consecuencias derivadas de la conducta inadecuada.

- Proponer diferentes opciones y que el alumno escoja aquella que prefiera, se castigue a sí mismo si es necesario.

- Bombardeo de ideas (batería de preguntas sobre lo expuesto en clase, preguntas selectivas de acuerdo con un nivel de competencia).

- Reflexión grupal sobre lo acontecido, pregunta al grupo: “¿qué pasa hoy?”.

Además de tener en cuenta estas estrategias para mejorar la gestión de clase creemos que es interesante conocer la opinión de los alumnos en cuanto a sus maestros. Para ello presentaremos las aportaciones de De Vicente (2006), obtenidas a partir de una consulta realizada a sus alumnos. Estas son las valoraciones que hacen de los profesores:

- Que el profesor sea auténtico, es decir, que exprese con sinceridad y eficacia lo que siente.

- Que el ambiente socioemocional del aula sea positivo de tal modo que cada alumno entienda que tiene un lugar adecuado y propio en el seno del grupo clase que le permita obtener éxito en la tarea que se propone.

- La reciprocidad, es decir que, aceptando los roles diferenciales entre profesor y alumno, las relaciones humanas en el aula estén basadas en la multidireccionalidad, la horizontalidad y simetría entre el profesorado y el alumnado.

- Contar con recursos para manejar las situaciones conflictivas tratando de no sobredimensionarlas.

- Que las expectativas sean positivas respecto a los alumnos y sus posibilidades de progreso.

- Que sea persuasivo en el sentido de transmitir una visión apasionada sobre aquello que se enseña.

- Favorecer la participación del alumnado en el aprendizaje, ya que en la medida, ya que en la medida que el alumno sea informado o puede influir sobre el programa docente, se establecerá un mayor nivel de vinculación del mismo con la tarea docente. 


\subsection{La metodología en el aula}

En este punto presentamos algunas propuestas metodológicas que se recogen en el artículo de Juan Carlos Torrego e Isabel Fernández "protocolo de actuación ante la disrupción en el aula" (p. 105) que hacen diferentes profesores para prevenir o disminuir las conductas disruptivas, son las siguientes.

- Refuerzos positivos (elogios verbales y de mirada, reconocimiento del trabajo, animo, proximidad corporal...).

- Llevar la clase organizada, llevar un guión ¿qué se va a trabajar? ¿con qué material? ¿cómo se va a evaluar?

- Adecuada organización del espacio para desarrollar la tarea programada.

- Utilizar los turnos de palabra para participar (aprender a escuchar).

- Cambio de actividad.

- Presión (exigencia de resultados y esfuerzo en la tarea), atracción (intercalar actividades distendidas), pausas (cambio de actividad).

- Diferentes formas de agrupaciones.

- Utilizar diferentes metodologías para atender a los diferentes estilos de aprendizaje.

- Supervisar y controlar posibles desajustes antes de que ocurran, estar muy atento.

- Promover el aprendizaje entre iguales y la cooperación.

- Utilizar diversas estrategias de evaluación.

\subsection{Actitudes preventivas del maestro}

Creemos que una buena práctica docente puede ser un elemento clave para evitar que los alumnos se desconecten de las clases y, como consecuencia tengas malas conductas en el aula. A continuación se plasman unas pautas tomadas de García (2008) que nos pueden ayudar a prevenir conductas disruptiva:

\section{PRINCIPIO DE LA CLASE}

Supervisión

Saludar, supervisar entrada.

Contacto visual.

Uso de nombres propios.

Uso de nosotros al hablar en grupo.

Dar impresiones verbales y no verbales tales como gestos, miradas, señales con el dedo.

Supervisar grupos de alumnos. 


\section{Tiempo-espacio}

Puntualidad.

Ocupa un lugar central, hacer comentarios precisos, mucho comentario visual.

\section{AL EMPEZAR LA TAREA}

Comenzar con la tarea individual de mesa.

Motivación

Activar la curiosidad e interés por el tema.

Hacer explícita al alumno la organización de la tarea (quién, qué, cómo, durante cuánto tiempo) y las metas que perseguimos.

Relacionar los contenidos con los intereses de los alumnos y con los conocimientos anteriores.

Hacer preguntas que ayuden a conectar la nueva información.

Contenidos

La programación debe contemplar tiempos y materiales, además de contenidos.

Hacer variedad de actividades y de estilos de aprendizaje: mirar, escuchar, hablar, escribir, preguntas y contestaciones, en parejas, en grupos.

Proporcionar tareas abiertas que admitan varias soluciones.

Estructurar la actividad de forma posible y comprensible.

Supervisión

Distribuir la atención sobre todos los alumnos.

Mantener una supervisión continua.

\section{DURANTE LA TAREA}

Motivación

Valorar las contribuciones creativas, incorporarlas si se puede.

Halagar-retroalimentar.

Mantener un ritmo, cambiar sin sobresaltos (las transiciones traen mucha disrupción).

No interrumpir el fluir de la clase innecesariamente, dejar para el final de las cosas de las que te acuerdes repentinamente.

Ayudar a los alumnos que lo requieren.

Contenidos-procedimientos

Aclarar dudas, preguntar por las dificultades.

No estar demasiado tiempo en un tema.

No tener prisa, respetar los tiempos individuales.

Utilizar estrategias que desarrollen temas. 
Algunos grupos necesitan distribuir la tarea entre los diferentes miembros, darles instrucciones o pautas específicas.

Tener preparadas tareas a diferentes niveles (atención a la diversidad).

\section{Supervisión}

No permitir que un grupo o una persona monopolice la atención del profesor, aconsejar, anotar, reprender, leer y escuchar en voz alta.

Ser consciente del espacio; delante, entre los pupitres, al final o moviéndose para ver las tareas de los alumnos.

\section{AL RECOGER Y SALIR}

Preparar y organizar el final de la clase: dejar tiempo.

Estar preparado para que los alumnos más conflictivos terminen antes que los demás la tarea.

Resumir lo que se ha hecho y conectarlo con los planes para el futuro.

La salida permite mantener una breves palabras con aquéllos/as que no hayan colaborado (p. 197).

\subsection{Creación de normas en el aula}

Uno de los principales elementos preventivos, será tener unas normas en clase que se deben explicar de una manera clara y concisa. Dependiendo del curso al que se esté impartiendo clase, sería interesante que sean los propios discentes los que elaboren las normas, ya que será mucho más motivador para ellos y serán más conscientes de lo que deben respetar ya que han sido ellos mismos las que las han redactado. Por supuesto esta labor debe estar supervisada siempre por el docente. Las normas de clase deben estar en un lugar visible dentro del aula para que los alumnos las vean y recuerden.

\section{La modificación de conducta}

Antes de pasar a explicar las diferentes técnicas de modificación de conducta vamos a detenernos en las definiciones que nos presentan algunos autores sobre el concepto de modificación de conducta.

Según Sampascual (2004) La modificación de conducta "se refiere al uso de la extinción para suprimir o eliminar las conductas no adaptadas y al uso del reforzamiento para incrementar las conductas deseadas" (p. 136).

Martin y Pear (2007) definen el término de la siguiente manera: La modificación de conducta implica la aplicación sistemática de los principios y las técnicas de aprendizaje para evaluar y mejorar los comportamientos encubiertos y manifiestos de las personas y facilitar así un funcionamiento favorable (p. 7). 
Una definición más actual es la hecha por Anguita (2010), que concluye lo siguiente:

"La modificación de conducta tiene como objetivo promover el cambio a través de técnicas de intervención psicológicas para mejorar el comportamiento de las personas de forma que desarrollen sus potencialidades y las oportunidades disponibles es su medio. El área de modificación de conducta es el diseño y aplicación de intervención psicológicas que permitan el control de la conducta para producir el bienestar, la satisfacción y la competencia personal" (p.2).

Después de ver las anteriores definiciones podemos concluir que la modificación de conducta es una intervención, que tiene como principal objetivo cambiar determinadas conductas en el individuo. Para ello se hace uso de una serie de estrategias que permitirán el control de las conductas que están perjudicando al individuo y a las personas que hay a su alrededor. Así la intervención con de las técnicas de conducta permitirán el bienestar y la mejora del sujeto.

Como es evidente, las conductas disruptivas en el aula no es algo que exista ahora, sino que ha existido siempre; es por lo tanto normal que los maestros hayan usado diferentes procedimientos para cambiar estas conductas, ya que como vinimos diciendo, producen la ruptura del proceso de enseñanza-aprendizaje.

Para llevar a cabo una intervención de modificación de conducta, podemos fundamentarnos en Skinner que tiene en cuenta una serie de elementos. Sampascual afirma que según los expertos los principales elementos son los siguientes:

- Definir el objetivo: En primer lugar, debemos establecer que conductas quiero eliminar o al menos reducir.

- Establecer la línea-base: Una vez determinadas las conductas a modificar, el siguiente punto será observar y registrar las conductas para establecer la línea-base, es decir, con qué frecuencia y duración se da la conducta que queremos modificar.

- Planificar la intervención y determinar las técnicas que se van a utilizar: Llegados a este punto lo que haremos será determinar qué técnicas son las más adecuadas para modificar las conductas.

- Evaluación de los resultados: En este punto reflejaremos los resultados que hemos obtenido una vez aplicado el programa de modificación de conducta.

\subsection{Técnicas de modificación de conducta}

Existen diferentes técnicas para modificar la conducta. A continuación vamos a ver qué tipos de técnicas podemos llevar a cabo en el aula, se clasificarán en dos grupos:

- Estrategias para el fomento de comportamientos adecuados.

- Estrategias para reducir y extinguir conductas inadecuadas. 


\subsubsection{Estrategias para el fomento de comportamientos adecuados}

\section{Imitación}

Es una técnica la podemos utilizar cuando queremos enseñar una nueva conducta al niño que este aprenderá por observación. Para ello habrá un modelo que ejecute la conducta que pretendemos que el sujeto adquiera; el modelo puede ser el propio maestro o compañeros. Valles Arándiga (1997) afirma que es importante que el modelo sea el idóneo para que el alumno pueda imitar su conducta, para ello se deben considerar algunas características importantes:

- Debe ser carismático o tener prestigio para el alumno.

- El modelo ha de ser sencillo para el alumno.

- Se deben premiar las conductas del modelo y también la imitación del alumno.

- La participación del alumno en el proceso debe ser activa.

- Todas las actividades deben ser reforzadas.

- Será de suma importancia tener en cuenta estas características a la hora de llevar a cabo esta técnica.

\section{Modelamiento}

Esta técnica consiste en que el sujeto desarrolle una nueva conducta, y éste será reforzado sucesivamente en cada pequeño paso que dé hacia la adquisición de la nueva conducta. El maestro expone detalladamente al alumno los pasos para llegar a la conducta-objetivo a conseguir, sin olvidar que tendrá que reforzar cada mínimo logro hasta conseguir la deseada. Esta técnica es muy útil, ya que el niño consigue adquirir nuevas conductas aprendiendo gradualmente lo que se espera de él, mientras se le va reforzando por sus pequeños avances. Podemos utilizar refuerzos verbales, para motivar al niño a conseguir la conducta deseada o refuerzos materiales. Para la aplicación de esta técnica es necesario tener muy clara la conducta que queremos lograr. El objetivo debe ser razonable y ser apropiado para el nivel evolutivo del niño/a.

Según Valles Arándiga (1997) el modelamiento de la conducta a adquirir:

Consiste en reforzar sistemáticamente cada pequeño paso o aproximación a la conducta terminal. Para aplicar esta técnica deberá hacerse una pormenorización o detalle de los pasos de que esta conducta la conducta-objetivo a conseguir para ir reforzando cada pequeña y gradual aproximación a la misma (p. 79). 


\subsubsection{Estrategias para reducir y extinguir conductas inadecuadas}

\section{Contratos de conducta}

Los contratos de conducta consisten en un acuerdo escrito entre las dos partes, en nuestro caso, alumno-profesor. El contrato es un reflejo del diálogo y negociación al que han llegado previamente maestro y alumno. En el contrato se determinarán las conductas que se quieren regular así como las consecuencias que tendrán para el alumno. Un contrato estaría compuesto de los siguientes aspectos:

- Datos personales del alumno y conductas-objetivo.

- Conductas específicas.

- Obtención de consecuencias.

- Otros (revisiones, canjes, modificaciones...).

- Firmas y fecha.

Jurado (2009) afirma que a la hora de aplicar esta técnica se debe tener en cuenta las siguientes pautas:

- El contrato debe ser negociado entre las partes que intervienen.

- Debe explicitarse las conductas en términos positivos.

- Deben especificarse las consecuencias positivas que obtendrá el alumno por la realización de las conductas, así como las consecuencias negativas por el incumplimiento.

- El contrato debe revisarse periódicamente.

- Está indicado para alumnos a los que se les supone cierta responsabilidad en su comportamiento (p. 3).

\section{Economía de fichas}

La economía de fichas es una técnica que fue desarrollada por Ayllon y Azrin en 1.968. Esta técnica fue usada por primera vez en hospitales psiquiátricos, su uso se extendió a instituciones de educación especial y finalmente se aplicó en las escuelas donde ha tenido una gran aceptación por la comunidad educativa. Pretende modificar las conductas indeseadas, para ello utilizará el refuerzo positivo cuando se emiten conductas deseadas y el castigo para las conductas indeseadas. El refuerzo positivo son fichas o puntos que se pueden cambiar por objetos de diferentes valores; mientras que el castigo consistirá en restar puntos que el alumno ya había conseguido por la buena conducta. Para llevar a cabo esta estrategia en primer lugar debemos determinar que conductas vamos a intentar eliminar y las escribiremos en una cartulina y las debemos poner en un lugar visible para que los niños las vean. Cuando el niño realice las conductas deseadas se le dará una pegatina verde por ejemplo y cuando haga una conducta indeseada se le dará una pegatina roja. Al final del día o de la semana (eso es una decisión de cada persona) se hará un recuento 
de los puntos que tiene el alumno y podrá sustituirlo por recompensas, que con anterioridad se hayan acordado entre alumno-profesor.

Antonio García (2008) afirma que los componentes básicos de una economía de fichas son los siguientes:

- Fichas que se puedan dar rápida y fácilmente.

- Un conjunto de reforzadores de apoyo.

- Reglas que especifiquen qué conductas ganan fichas y las fichas que se pueden ganar

- Reglas que especifiquen cómo se pueden gastar o cambiar las fichas.

- Los procedimientos para ir cambiando reglas para el reforzamiento conforme se van produciendo el progreso.

- Los procedimientos para quitar el sistema de fichas al mismo tiempo que se mantiene el orden, respeto y convivencia (p.23).

\section{El castigo}

Según el Diccionario de la RAE, castigo se define como "la pena que se impone a quien ha cometido un delito o falta." Podemos decir, por lo tanto, que castigo es la corrección que haremos ante una conducta inadecuada con el objetivo de modificar dicha conducta.

Ante la conducta indeseada del niño le presentaremos a éste, algo desagradable o le quitamos algo agradable. Una de las ventajas de esta técnica es su efecto inmediato; aunque también tiene algunas desventajas como que puede producir miedo o temor, que el niño aprenda a huir o escurrir el bulto para escapar del castigo y que normalmente el efecto del castigo dura muy poco y no cambia esa conducta de forma permanente.

\section{Extinción}

Consiste en retirar la atención a una conducta no deseada, es decir, ignorar las conductas inadecuadas del niño. Vallés Arándiga (1997) afirma que la extinción consiste, pues, en ignorar la conducta anómala, pero paralelamente es conveniente reforzar la conducta opuesta, prestarle atención a la conducta deseada para aumentar la probabilidad de su ocurrencia.

\section{Aislamiento}

Consiste en retirar unos instantes del lugar o situación donde se está produciendo la conducta que pretendemos eliminar, castigando de este modo la conducta inadecuada. En el ámbito educativo aplicar esta técnica plantea un problema y es la elección de aislamiento social (pasillo, patio...). 
Vallés Arándiga (1997) afirma que: El aislamiento consiste en separar contingentemente al niño del contexto en el que está ocurriendo la conducta anómala (p.105).

Algunas conclusiones a las que han llegado Patterson y White con respecto a la eficacia de la técnica del aislamiento son:

- La técnica de aislamiento suele ser más eficaz que adoptar una actitud pasiva ante la conducta-problema.

- El tiempo fuera no debería rebasar los 20 minutos.

- Si la conducta-problema aumenta al ordenar al niño salir de clase, debe aplicarse un castigo moderado en función de la persistencia del problema.

\section{Conclusiones}

Las conductas disruptivas son un fenómeno que afecta gravemente al proceso de enseñanza-aprendizaje no permitiendo al maestro desarrollar la clase y provocando así un clima negativo y hostil en el aula.

El docente que tenga alumnos con conductas disruptivas en el aula, debede analizar la situación personal y familiar del alumno para poder ayudarlo. Solamente conociendo todo lo que le rodea podrá llevar a cabo una buena intervención educativa para solucionar el problema.

Es necesario tener en cuenta, que no solo afectan a la conducta del alumno los factores externos, sino que también los factores internos del centro; por lo tanto, el docente tiene un papel fundamental para evitar conductas disruptivas en el aula.Existen numerosas estrategias y técnicas de modificación de conducta que serán de gran utilidad para cambiar las conductas disruptivas del alumno por un buen comportamiento.

\section{Referencias}

ANGUITA, J. M. Modificación de conducta. Innovación y experiencias educativas, n. 35, 2010.

CASTEJÓN, J. L.; NAVAS, L. Aprendizaje, desarrollo y disfunciones: implicaciones para la enseñanza en la educación secundaria. Alicante: Editorial Club Universitario, 2009.

DE VICENTE en TORREGO, J. C., FERNÁNDEZ, I. La disrupción y la gestión en el aula. Convivencia en la escuela, v. 3, p. 1-8, 2006. GARCÍA. A. La disciplina escolar. Murcia: Universidad de Murcia. 2008.

GOTZENS, C. (1986) en GARCÍA. A. La disciplina escolar. Murcia: Universidad de Murcia, 2008.

FERNÁNDEZ, F. Inventario de hábitos de estudio: Manual. Barcelona: TEA, 2002.

Educação Por Escrito, Porto Alegre, v. 8, n. 2, p. 278-293, jul.-dez. 2017 
FERNÁNDEZ, I. Haciendo frente a la disrupción desde la gestión del aula. Modelo de mejora de la convivencia; estrategias de mediación y tratamiento de conflictos. Barcelona: Graó, 2006.

GARCÍA, A. Disciplina escolar: Guía docente. Murcia: Universidad de Murcia, 2008.

GARRY, M.; PEAR, J. Modificación de conducta, qué es y cómo aplicarla. Madrid: Pearson Education, 2008.

GÓMEZ, M. Los sistemas de ayuda entre iguales como instrumentos de mejora de la convivencia escolar: evaluación de una intervención. Madrid: MECD, 2009.

HOLLINS, T. Teacher's attitudes to children's behavior. Manchester: Ed. University of Manchesterm, 1955.

JURADO, R. Técnicas para la instauración y/o disminución de conducta. Innovación y experiencias educativas, v. 16, 2009.

LATORRE, A.; TERUEL, J. Protocolo de actuación ante conductas disruptivas. Proaxi, v. 95, p. 62-74, 2009.

MARTÍN, G.; PEAR, J. Modificación de conducta. Qué es y cómo aplicarla. Madrid:Pearson; 2007

PELECHANO, V. Terapia y modificación de conducta. Fundación Juan March, 1979.

SAMPASCUAL, G. Psicología de la educación. Madrid: UNED, 2004.

TORREGO, J. C.; FERNÁNDEZ, I. La disrupción y la gestión en el aula. Convivencia en la escuela, v. 3, p. 1-8. 2006.

. Protocolo de actuación ante la disrupción en el aula. Proyecto Atlántida, p. 93-110. 2007.

URUÑUELA, P. Convivencia y conflictividad en las aulas: análisis conceptual. Disrupción en las aulas problemas y soluciones. Madrid: MECD, 2006.

VALLÉS, A. Modificación de la conducta problemática del alumno, técnicas y programas. Alicante: Marfil, 1997. Modificación de la conducta problemática del alumno, técnicas y programas. Alicante: Marfil, 2002.

Recebido em: julho/2017

Aceito em: novembro/2017

Endereço para correspondência:

Ctra. Ledrada 1, 37791 Peromingo

Salamanca, España

<mgomezgo@yahoo.es> 